\title{
An Optimization Strategy for Improving the road Emergency Guarantee System in Hubei Province
}

\author{
ZihanZeng ${ }^{1, a}$ \\ ${ }^{1}$ School of Politics and Administration, Wuhan University of Technology, Wuhan, P.R.China, 430070 \\ azengzihan1111@163.com
}

\begin{abstract}
Keyword: emergency guarantee system; geological disaster; 5-factor framework; system optimization Abstract: A road emergency guarantee system is a prerequisite for ensuring that emergency relief activities, such as the transportation of emergency supplies or the deployment of medical emergency teams, can proceed smoothly. In this paper the classic 4R model developed by Robert Hess and the 5-factor model are combined, and a theoretical framework with both horizontal and vertical dimensions is established. Moreover, on the basis of the developed framework and specific cases, the road emergency guarantee system in Hubei province is further evaluated, the existing research and analytic results are integrated, and an optimization strategy regarding the three major phases (reduction, readiness and response) of the road emergency guarantee system in Hubei province is proposed. Given that the current study is based on the actual situation in Hubei province, it could provide a valuable reference for optimizing the road emergency guarantee system in the area.
\end{abstract}

\section{Introduction}

Hubei province is located in the middle reaches of the Yangtze River, and its terrain decreases in elevation from the west to the east. The eastern, northern and western regions are surrounded by mountains, whereas the central and southern regions are at a low elevation. According to the elevation gradient and morphological characteristics, the terrain of Hubei province can be divided into four basic types; namely, mountains, hills, mound land and the plain, among which the mountainous regions (i.e., the western, northwestern and southwestern regions of Hubei province) are vulnerable to mountain collapses and landslides. The extensive development of mudslides makes such regions well-known for geological disasters, leads to a huge loss of life and property, and severely endangers road safety. Due to the rapid development of China's economy, road construction in Hubei province is being extended to regions that have suffered severe geological disasters. Under the circumstances of sudden yet unexpected disasters, the road emergency guarantee system is a prerequisite for ensuring that emergency relief activities, such as the transportation of emergency supplies and human resources, can proceed smoothly. The extant research on the establishment of an emergency response system tends to put an emphasis on the possibility of a universal application that covers a wide range of situations, but neglects the specific and unique nature of an emergency guarantee system which is context-dependent. Therefore, theoretical research on the development of an emergency guarantee system that is suitable for the type of natural disasters in Hubei province is an important topic in the current research, and is of practical significance and significant value.

\section{The establishment of a theoretical framework}

The 5-factor theoretical framework.It can be seen from the construction and development of the road emergency guarantee systems in the US, Europe, Japan and other developed countries that a comprehensive system of this nature should include five major elements: emergency team, emergency equipment, emergency supplies, contingency plan and emergency fund. Moreover, it is important to have these elements effectively coordinated and organized so that rescue resources can be maximized or optimized, and emergency response capabilities reinforced. 
An emergency team refers to a group of people, ranging from road inspectors and road maintenance workers to on-site technicians and road administration officers, who coordinate to deal with road emergencies. Emergency equipment refers to the mechanical equipment required, ranging from pre-disaster prevention to post-disaster reconstruction, and information equipment such as a traffic monitoring system. Unlike ordinary materials, emergency supplies are time-sensitive and irreplaceable and according to their purpose could be further divided into 13 categories, including equipment and tools, engineering equipment, lighting equipment, power fuels, traffic and transportation and engineering materials. A contingency plan refers to a pre-prepared comprehensive or specific management plan that deals with possible disasters. It should be noted that this paper specifically focuses on the discussion of natural disasters, with the characteristics of Hubei province taken into account. An emergency fund refers to the money used to deal with emergency management, which is collected through government guidance and social participation. In summary, these five elements constitute the assessment framework of the emergency guarantee system in a horizontal arrangement.

The 4R emergency management framework.Another widely recognized disaster management cycle model is based on the time dimension, namely the classic 4R model developed by Robert Hess; with the 4 Rs referring to Reduction, Readiness, Response and Recovery. Reduction refers to decreasing the risks of a disaster or the possibility of a disaster occurring, from both policy and facility perspectives, with a clear understanding of the location of risk sources. Readiness refers to the integration of pre-disaster warnings and related contingency plans. Response refers to the rescue, assistance, and maintenance work after the disaster has occurred. Finally, recovery refers to a series of rehabilitation and reconstruction works in disaster-hit areas.

It can be seen that although the content of the emergency guarantee system is relatively complicated, the four phases, reduction, readiness, response and recovery, represent the fixed components in a vertical arrangement. In this paper, the emergency guarantee system discussed mainly consists of the first three phases, whereas the last phase, recovery, is not within the scope of the present study.

\section{A current situation analysis based on the theoretical framework}

China has established a relatively comprehensive road emergency guarantee system, which is based on government and department emergency platforms, with road emergency stockpile centers as the connected nodes. However, when compared with some developed countries, China's emergency response capability is still in its infancy. For example, if one considers the construction requirements of an emergency road transportation system, the current progress in China is still lagging behind. Moreover, if any severe natural disasters were to occur (despite such events taking decades or even centuries to take place), the current emergency guarantee system would not be sufficient to deal with them.

The current state of the road emergency guarantee system.In Hubei province, the main problems of the current emergency guarantee system are more acute in certain economically underdeveloped areas, such as the western, northwestern and southwestern regions of the province. These regions have been constrained by economic conditions for decades, and policy makers within them are making great efforts to increase their wealth. However, they often tend to overlook the importance of constructing and improving their road emergency guarantee systems. Against the background of economic development, these urbanized pioneer regions are often very vulnerable to natural disasters (mainly in the form of geological disasters). Therefore, the disadvantages of the current emergency guarantee system include: the low level of emergency response and organizing capabilities, and a limited number of on-site aides and social workers; the lack of a compensation mechanism for emergency guarantees and emergency requisition purposes; weak provision for emergency technical support, as well as poor coordination and communication across the regions; the lack of risk assessment in road traffic plans, 
and a lack of dynamic information and scientific support; weak emergency guarantee skills and a lack of practice at the local level; the lack of professional training for emergency teams; and the insufficiency of emergency supplies.

Case analysis .If two of the typical disaster areas in Hubei, Enshi and Lichuan, are taken as examples, the survey data show that the builders, highway personnel and security management staff of the highway emergency system in Enshi are clearly insufficient, with an average level reaching about $66.7 \%$ of the demand. Furthermore, there are big gaps in the personnel numbers of the mechanical operating and social forces. The emergency mobilization is fairly passive and the linkage between the responsibility zone and the mobilization of social volunteers does not meet the requirements of the current emergency situation effectively.

Lichuan is located in the hilly area of southwest Hubei, which is an area with a high traffic accident rate. Statistics show that the emergency funds of southwest and northwest Hubei cannot satisfy the demand caused by emergencies. The funding gap of Lichuan alone is as much as RMB 1.5 million, where the main areas of focus are the lease, repair and maintenance of the emergency support system's equipment, the purchase of supplies and the payment of temporary workers' wages. At the same time, in southwest and northwest Hubei, the task of repairing sections of the road following serious highway disasters is very challenging and the special repair fund is wholly insufficient to meet current needs. For example, the funding gap for the dangerous crags at the top left-hand section of the G242 Gan-Qin highway (which includes K39+380-K40+000 of the former S248 Li-Zhi highway) in Lichuan is RMB 9.3 million. Furthermore, there are 25 super disaster restoration projects that require RMB 283.12 million for successful completion. Therefore, from the perspective of those who wish to prevent disasters, the present emergency fund mechanism cannot meet the level of demand.1

There are defects and lack of operability issues regarding the planning system in each region. The existing plans are mainly focused on single disasters (such as snowstorms and earthquakes), and thus unable to effectively respond to the concurrent occurrences of multiple disasters. Moreover, the implementation status of plans is generally poor according to the survey. After the emergency response law of the People's Republic of China decreed that the "emergency response should mainly follow the rule of prevention-oriented and the combination of prevention and emergency", the problem of superficial work arises: that is planning for the plan, which is reflected in the survey data by the fact that emergency plans of different counties and cities are similar. This indicates that current emergency plans are of low operability, poorly implemented and lead to difficulties in responding to comprehensive and concurrent disasters. Moreover, the statistics show that the drills of most local emergency response departments cannot meet current demands and some regional areas have no annual drills at all.

\section{An optimization strategy for the improvement of the road emergency guarantee system}

The two models with their vertical and horizontal arrangements have covered most parts of the road emergency guarantee system. Following a full consideration of the characteristics of natural disasters in Hubei province and the advanced practices of the US, European countries and Japan, this paper makes the following recommendations based on the two theoretical frameworks.

The reduction phase.The disaster emergency management system in Japan places a high emphasis on the "equal treatment of times of peace and emergency" and regards the notion of "nipping something in the bud" as important in an emergency. Japan treats the development of a traffic emergency support system as a critical object to implement. This includes the pre-disaster stability evaluation of infrastructure, the collection of information resources, and peacetime emergency drills. Under such a system, highway related departments in Hubei should carry out pre-evaluations on sources of disasters, such as mountain collapses, landslides, debris flow, and ground collapses as part of its highway 
emergency support system. Specifically, areas where there is the potential for severe disasters, such as west Hubei, southwest Hubei, northwest Hubei and other areas where there is a high incidence of natural disasters, should put place a greater emphasis on the development of robust and widespread monitoring and prevention mechanisms.

With regard to teams, it is necessary to establish a professional exploration assessment team, periodically inspect and evaluate the stability of each road section and collect road information. Moreover, excellent emergency teams need to be established, particularly emergency reopening teams and supply transportation support teams. In advanced western countries such as Germany, the establishment of emergency response teams has many features, which could be summarized as five "combinations": the combination of rescue and relief; the combination of comprehension and profession; the combination of full-time and part-time; the combination of government and society; and the combination of locality and federation. The specific application to Hubei province is mainly reflected in four aspects: establishing professional emergency response teams according to the local risk evaluation; keeping the comprehensive emergency response teams in an armed state; uniting social forces as a possible way of establishing professional emergency response teams (i.e. using volunteers as the main force); and enhancing training by imparting rescue skills and providing practical exercises.

With regard to equipment, it is necessary to realize the interregional integration and management of equipment and build a management system of emergency equipment using information technologies. Furthermore, emergency equipment needs to be encoded and registered using a national emergency response platform and it must be ensured that such platforms can collate information and share resources. An emergency equipment management system should aim for the goal of "close to the demand, deepen the application" and enhance the equipment management requirements of scientization, intelligentization and standardization to improve the emergency response capability.

With regard to supplies, a targeted reserve of supplies needs to be established, periodic checks conducted and emergency supplies supplemented in the regions that are evaluated as poor with regard to stability. For the site selection of emergency supplies, the drivers of emergency vehicles should be surveyed to obtain the index weights of road conditions and a fuzzy evaluation applied to determine the optimal location plan; this ensures that emergency supplies take on the role of an emergency response in the first stage of the disaster.

The readiness phase.Only when a good foundation is built in the readiness phase can the following emergency response and management work be conducted in an orderly manner. This phase includes two major components: the emergency contingency planning mechanism and emergency response warning mechanism. The core of contingency planning is to build a relatively complete framework for resource coordination, staff arrangements and workflow allocation that allows for the circumstances of emergency situations. Through a series of detailed provisions and practical measures, the disorder and chaos caused by such emergency situations can be reduced or eliminated as much as possible. Meanwhile, the purpose of emergency warnings is to provide some scientific forecasting and assessment based on collected road traffic data or road section information, so that the demands of a road emergency rescue can be met in a timely manner.

Contingency planning practice, as far as the characteristics of natural disasters in Hubei province are concerned, should be based on the extensive and scientific risk assessments conducted in the reduction phase. For example, the published data of the Department of Road Administration of Lichuan city showed that along the G350 Lichuan-Luhuo line (previously known as the Lichuan-Yulong line, K36+350-K37+020 sections), unstable rocks on the right upper slope have posed potential risks. Therefore, countermeasures and suggestions for dealing with such risks should be put forward in emergency planning practice. 1

With regard to emergency equipment, the EPC System Network and ITS Intelligent Transportation

${ }^{1}$ Data are from onsite survey 
Technology are needed to achieve the goals of overall control, unified command, monitoring and management, intelligent scheduling, emergency handling, security control, and information management of work behavior. Through a comprehensive analysis of the number of working vehicles, their distribution patterns, the intact rate and the amount of consumed supplies, the emergency response work can be optimized in order to improve on-site rescue efficiency.

With regard to the emergency fund, the main sources of the US emergency fund are the annual US federal budget, US federal emergency guarantee fund, presidential disaster relief fund, as well as the presidential and congress granted special fund. In Japan, the central and local government are responsible for $27 \%$ and $73 \%$, respectively, of recovery and reconstruction expenses following natural disasters. This leads to the conclusion that when preparing an annual budget, a government should set aside funding to deal with unexpected disasters. Therefore, a government should set up a reserve to ensure that cost overruns caused by unexpected disasters won't lead to a budget deficit. Additionally, a compulsory insurance system should be established with government intervention, with the insurance being made compulsory on the basis of a unified rate control.

The response phase.The response phase includes the early disposal, reporting and assessment of disasters, initialization of contingency plans, emergency rescue and assistance, as well as on-site organization and maintenance. On the basis of the reduction and readiness phases, the priority is put on the pre-treatment of disaster-hit roads. Through scientific disaster assessment and analysis, the type of contingency planning required is determined and activated. Subsequently, and according to the developed plan, emergency teams are dispatched immediately, and the linkage between all levels of command and coordination is strengthened.

With regard to emergency supplies, since most of these are stored in road emergency stockpile centers, the emergency response phase requires urgent mobilization of available resources. The type and amount of required emergency supplies in disaster-hit road sections is determined on the basis of a pre-disaster assessment. Moreover, by using the results of fuzzy clustering analysis, disaster-hit road sections are grouped. The fuzzy multiple objective programming method is used to establish the optimization model, and thus find the optimal route for delivering supplies so that emergency supplies can reach disaster sites for rescue purposes as soon as possible. Meanwhile, interactive responses should be used to send emergency supplies to different regions; therefore when one disaster-hit road section is temporarily out of supplies, upper-level emergency stockpile centers should be able to mobilize available resources from other regions in a timely manner.

With regard to contingency planning, the decision to activate a contingency plan or multiple contingency plans depends on a quick assessment of the aftermath of a disaster. At the same time, a brief report should be delivered quickly to the upper-level emergency guarantee agency based on the level of damage. In order to ensure that a contingency plan has sound operability, high flexibility and low costs, the initialization of such a plan should be conducted in different stages.

\section{Conclusions}

Due to the fact thatnatural disasters can occur in very complicated ways, simple static models no longer meet the needs of the road emergency guarantee system of Hubei province. Consequently, the establishment of a road emergency response system should be based on an assessment of dynamic risks and stability, with a priority put on the five aspects (emergency teams, emergency equipment, emergency supplies, the contingency plan and emergency fund) and three dimensions (reduction, readiness and response) so that the developed system can adapt effectively to changing emergency situations. 


\section{References}

[1] Liu Chengwu, Huang Limin, Wu Binxiang.Discussion on impacts of man-land relationship on natural disaster in Hubei province [J]. Research of Soil and Water Conservation, 2004 11(1):177-181.

[2] Li Jing, An Shi, Cui Jianxun. Road traffic emergency management research [J]. Journal of Harbin Institute of Technology (HIT, Social Sciences Edition), 2009(6):139-145.

[3] Li Fuzhong. Research on the establishment of regional traffic emergency guarantee system [D].Southwest Jiaotong University, 2012.

[4] Haddow G D, Bullock J A, Coppola D P. Introduction to emergency guarantee[J]. Journal of Homeland Security \& Emergency guarantee, 2011(3).

[5] Tong Xing, Tao Peng. The innovation of emergency management system in China--relevant perspectives based on source management, dynamic management and emergency guarantee [J]. Jianghai Academic Journal, 2013(2):111-117.

[6] Xiao Runmou, Yan Shengyi. The assessment of the selection of highway emergency supplies storage sites using multi-traffic indexes [J]. Journal of Safety and Environment. 2015(3):53-57.

[7] Jiang Bin, Long Haifeng, Li Ming. Regional dynamic working of maintenance machinery and application of emergency guarantee system [J]. Road Machinery \& Construction Mechanization, 2015(5):40-44.

[8] Zhao Shangmei, Yang Xuemei. The funding safeguard mechanism of public emergencies in China --a theoretical framework [J]. Chinese Public Administration, 2012(12):44-47.

[9] Chen Yizhou, Yang Rui, Su Guofeng. Study on technologies for classifying and managing emergency equipment resources [J]. China Safety Science Journal, 2014(7):166-171.

[10] Zhang Lei. The construction of emergency rescue teams: the German model and reference. Journal of Chinese Academy of Governance, 2012(3):39-42.

[11] Yang Saini, Ma Weifeng, Liu Hao. Road emergency logistics distribution under earthquake-induced secondary landslide disaster [J]. Journal of Highway and Transportation Research and Development, 2011, 28(7):152-158.

[12]Yu Hui, Jiang Zhihui. Study of the model of activating contingency plans within disruptive accidents by stages [J]. Journal of Industrial Engineering and Engineering Management, 2011, 25(1):109-114. 